\title{
Modeling Hydraulic Accumulators for use in Wind Turbines
}

\author{
Henrik Brun Hansen and Peter Windfeld Rasmussen \\ R\&D/Fritz Schur Energy, Glostrup, Denmark \\ E-mail: hbh@fsenergy.dk, pwr@fsenergy.dk
}

\begin{abstract}
One of the major challenges in numerical simulation of hydraulic systems, is the long computation times of accumulators. In wind power applications, the accumulators must provide necessary hydraulic energy during emergency stops, while the weight of hydraulic equipment must be minimized. Therefore, precise and efficient design tools for accumulators are essential.

This paper addresses the issue of understanding the dynamic phenomena in piston type accumulators, and how this leads to improved numerical accumulator models. Developing a numerical model has two challenges. First, the unsteady heat transfer between the accumulator gas and the wall has to be described. Secondly, a suitable real gas model has to be identified and employed. To verify the model, a series of experiments were conducted at Fritz Schur Energy in Glostrup, Denmark. The experiments were designed to investigate gas dynamical properties at various precharge pressures, maximum pressures, and ambient temperatures. These parameters were varied to obtain parameter independent conclusions. During controlled piston movements, hydraulic and gas pressures were measured together with the piston position and the gas temperature.

It was found that the simple thermal time constant approximation by Rotthäuser was suitable and stable for the application. It was also found that the Soave-Redlich-Kwong equation was overall best suited with experimental data. The Soave-Redlich-Kwong equation is as much as six times faster than the widely used Benedict-Webb-Rubin equation, independent of the ambient temperature, maximum pressure and precharge pressure.

This project concludes that the Soave-Redlich-Kwong equation should be used in simulation of piston type accumulators. It is noted that the pressure available after expansion is some 5-10 bar lower than predicted by any model. Therefore, it is suggested that more research is conducted to obtain an improved model for the heat transfer. Finally, measurement quality was confirmed by comparing measured pressure data by pressure calculations based on measured temperature.
\end{abstract}

Keywords: Hydraulics, Accumulator, Thermodynamics, Modelling, SIMULINK, Matlab, Verification, Thermal time constant

\section{Problem Introduction}

Hydraulic pitch systems in wind turbines require a reliable safety system to enable emergency stop without the presence of electrical power. Figure 1 shows the general system layout for each blade.

The system must supply volumes in the range 10 to 25 liters of oil in less than 10 seconds for each blade. Hence accurate calculation of the accumulator size in a temperature range from $-30^{\circ} \mathrm{C}$ to $+50^{\circ} \mathrm{C}$ is essential.

\subsection{Motivation}

Over the years a number of models have been presented and tested. Otis et al presented the Benedict-Webb-Rubin (BWR) model in 1985 [1], as an expansion of the Beatie-Bridgeman
(BB) model from 1974 [4]. However practical experience has shown that these models have limited accuracy at low temperatures. Another aspect in modern computational analysis is the time required to run the simulation. Debugging the system model shows that the accumulator model is the part requiring the smallest time steps. Hence reducing the simulation time will require a faster numerical model of the accumulator.

\section{Theory}

In order to model the phenomena in the accumulator gas, a thermodynamical model is set up using the first law of thermodynamics:

$$
\dot{U}=\dot{Q}_{c o n}-\dot{W}
$$




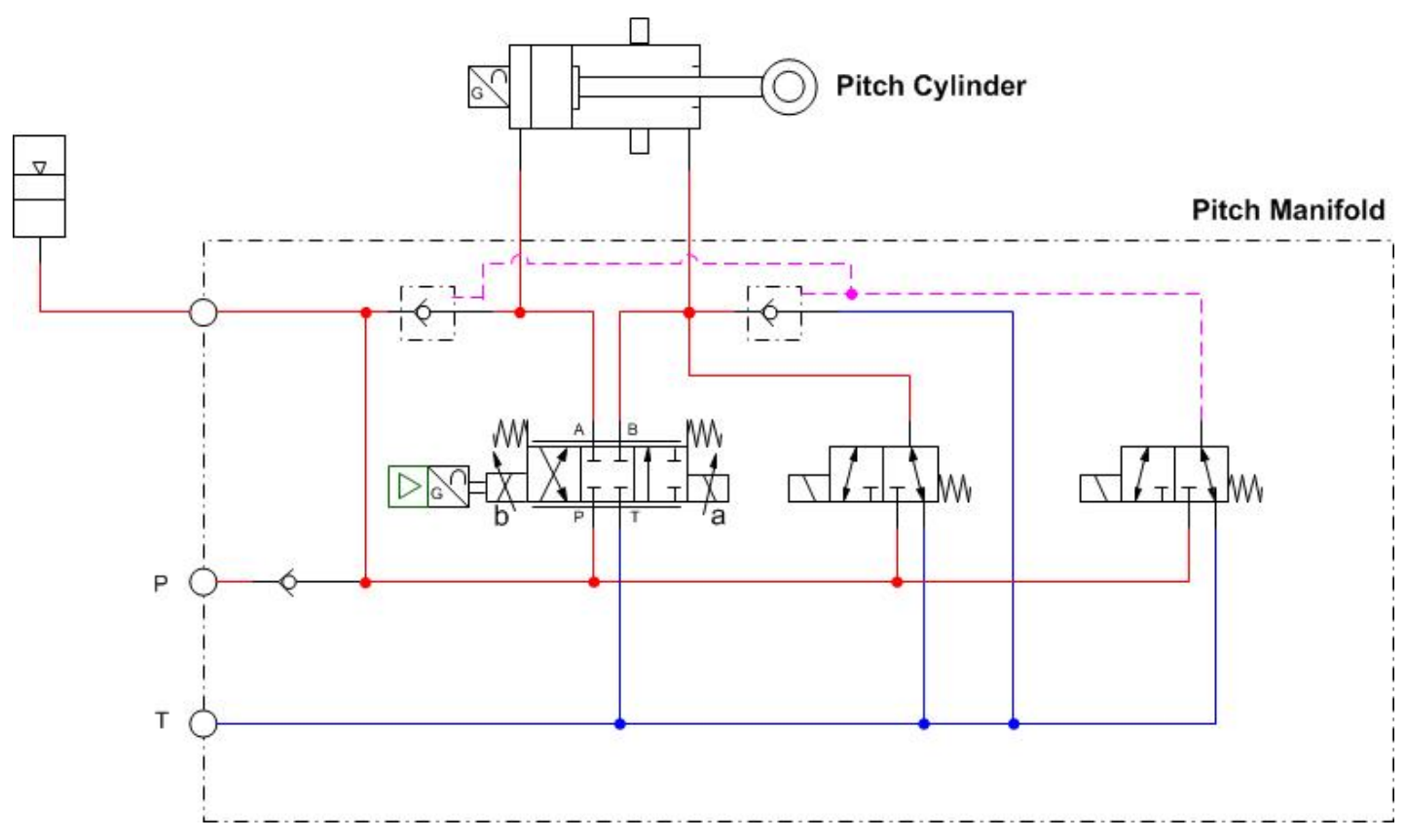

Figure 1: The system layout for each wind turbine blade

All symbols are explained in the nonmenclature (table 1 page 3). This is a simple energy balance on the gas. Consider the differential equation by Otis [2]:

$$
\dot{U}=m c_{v} \frac{d T}{d t}+m\left(T\left(\frac{\partial p}{\partial T}\right)_{v}-p\right) \frac{d V}{d t}
$$

Since:

$$
\dot{W} \equiv p \frac{d V}{d t}
$$

and the heat transfer from a system can be written

$$
\dot{Q}_{\text {con }}=h A\left(T_{a}-T\right)
$$

Combining equation 1, 2, 3 and 4 yields:

$$
\begin{gathered}
h A\left(T_{a}-T\right)-p \frac{d V}{d t}=m c_{v} \frac{d T}{d t}+ \\
m_{\text {gas }}\left(T\left(\frac{\partial p_{\text {gas }}}{\partial T}\right)_{v}-p_{\text {gas }}\right) \frac{d V}{d t}
\end{gathered}
$$

Which is a first order, heterogeneous partial differential equation. It can be simplified to:

$$
\frac{d T}{d t}=\frac{h A}{c_{v} m}\left(T_{a}-T\right)-\frac{m T}{m c_{v}}\left(\frac{\partial p_{g a s}}{\partial T}\right)_{v} \frac{d v}{d t}
$$

This equation can further be simplified by introducing the thermal time constant, $\tau$, which can be written in the form:

$$
\tau=\frac{m_{g a s} c_{v}}{h A}
$$

Then equation 6 becomes:

$$
T=\frac{\left(T_{a}-T\right)}{\tau}-\frac{T}{c_{v}}\left(\frac{\partial p_{\text {gas }}}{\partial T}\right)_{v} d v
$$

This differential equation can be solved when combined with some equation of state for the accumulator gas. The equation should express gas pressure as a function of temperature, volume and gas specific constants, as:

$$
p=\mathscr{F}\left(T, V, \mathbb{C}_{1}, \mathbb{C}_{2}, \ldots, \mathbb{C}_{n}\right)
$$

By taking the partial derivative of the equation of state, it can be inserted into equation 8 .

$$
T=\frac{\left(T_{a}-T\right)}{\tau}-\frac{T}{c_{v}}\left(\frac{\partial}{\partial T} \mathscr{F}\left(T, V, \mathbb{C}_{1}, \mathbb{C}_{2}, \ldots, \mathbb{C}_{n}\right)\right)_{v} d v
$$

The problem of determining the gas temperature has now reduced significantly. When the change of gas temperature from a small volume change, $d v$, is known, it can be substituted back into the chosen equation of state to determine the pressure change. By doing this, the pressure and temperature development in the accumulator gas can be determined accurately. However, two questions still remain:

1. How to determine the thermal time constant, $\tau$ ?

2. Which equation of state should be chosen?

These questions frame the main challenge of this article.

\subsection{The Thermal Time Constant}

To answer the question regarding the thermal time constant, it is necessary to physically understand it. As seen in equation 7. it depends on the heat transfer coefficient, $h$, gas properties and the exposed wall area, $A$. It therefore expresses something about the time it takes for the gas to transfer heat to the accumulator wall. It can be expanded by writing out the heat transfer coefficient:

$$
h \equiv \frac{Q}{A \Delta T}
$$




\begin{tabular}{|llr|}
\hline Symbol & Explanation & Unit \\
\hline$\dot{U}$ & Rate of internal energy change & $\frac{J}{s}$ \\
$\dot{Q}_{C o n}$ & Rate of convective heat transfer & $\frac{J}{s}$ \\
$Q$ & Heat flow & $\frac{J}{s}$ \\
$\dot{W}$ & Rate of work done & $\frac{J}{s}$ \\
$m$ & Gas mass & $\mathrm{kg}$ \\
$T$ & Gas temperature & $\mathrm{K}$ \\
$t$ & Time & $\mathrm{s}$ \\
$p$ & Gas pressure & Pa (bar when noted) \\
$V$ & Gas volume & $m^{3}$ \\
$h$ & Heat transfer coefficient & $\frac{W}{m^{2} K}$ \\
$A$ & Exposed wall area & $m^{2}$ \\
$T_{a}$ & Ambient temperature & $\mathrm{K}$ \\
$c_{v}$ & Specific heat capacity at constant volume & $J$ \\
$\tau$ & Thermal time constant & ${ }^{k g K}$ \\
$\mathbb{C}$ & Some constant & $\mathrm{s}$ \\
$v$ & Specific volume & Somit \\
$R$ & The gas constant & $\frac{m^{3}}{k g}$ \\
$T_{c}$ & Critical temperature & $J$ \\
$p_{c}$ & Critical pressure & $\mathrm{K}$ \\
$\omega$ & Acentric factor & $\mathrm{K}$ \\
$P_{0}$ & Preload pressure & $\mathrm{Pa}$ \\
$T_{0}$ & Preload (initial) temperature & - \\
$A_{0}, a, B_{0}, b, C_{0}, c$ & Benedict-Webb-Rubin equation constants & ${ }^{\circ} \mathrm{C}$ \\
\hline & & - \\
\hline
\end{tabular}

Table 1: Nonmenclature

By inserting into equation $7, \tau$ becomes:

$$
\tau=\frac{m_{\text {gas }} c_{v}}{\frac{Q}{A \Delta T} A}=\frac{m_{\text {gas }} c_{v} \Delta T}{Q}
$$

In many applications, $\tau$ is constant since the heat flux can be assumed constant. Therefore $\tau$ is called a thermal time constant. However, as indicated by [2], it is not constant when examining accumulator dynamics. It must therefore be evaluated as gas temperature and heat flow changes. Equation 12 does not offer easier computations than equation 4 It merely shows that $\tau$ is independent of exposed wall area. It solves the problem of finding the heat transfer coefficient between the accumulator gas and the accumulator wall, but instead it introduces the heat flow. In hydraulics, this property is often unknown. However, much effort has been put into approximating $\tau$, for example [2] and [3]. More and less complicated approximations have been presented. For the scope of this research, the simple approximation by [3] proved sufficient:

$$
\tau \approx 0.3 p V^{0.33}+86.2 V^{0.49}
$$

Note: Only for piston type accumulators.
Even though $\tau$ physically varies through compression or expansion, the results obtained when applying the presented thermodynamical model, equation 8 , are not compromised much by assuming a constant $\tau$. This is also noted by [4].

\subsection{Equation of State}

The subject of choosing the equation of state is the main contribution of this article. Parallel models employing equation 8 with different equations of state were made and compared to experimental data.

- The Van Der Waals equation

- The Beatie-Bridgeman equation

- The Benedict-Webb-Rubin equation

- The Redlich Kwong extension to the Van Der Waals equation

- The Soave extension to the Redlich Kwong extension to the Van Der Waals equation (The Soave-Redlich-Kwong equation) 
However, since the Benedict-Webb-Rubin equation is an improvement of the Beatie-Bridgeman equation, and since the Soave-Redlich-Kwong equation is an improvement of the other Van Der Waals based equations, the analysis can be reduced to the comparison between the two. They are explained in the following.

\subsubsection{The Benedict-Webb-Rubin Equation}

This model offers greater complexity than the others. It is like the Beatie-Bridgeman equation based on empirical data and it is widely recognized for being very accurate. It can be written:

$$
\begin{array}{r}
p=\frac{R T}{V}+\frac{B_{0} R T-A_{0}-\frac{C_{0}}{T^{2}}}{V^{2}}+\frac{b R T-a}{V^{3}}+\frac{a \alpha}{V^{6}}+ \\
\frac{c\left(1+\frac{\gamma}{V^{2}}\right) e^{-\frac{\gamma}{V^{2}}}}{V^{3} T^{2}}
\end{array}
$$

\subsubsection{The Soave-Redlich-Kwong equation}

Soave [5] improved the Redlich-Kwong equation by introducing the acentric factor, $\omega$. In short, the acentric factor is a measure of how non-spherical a molecule is. It thereby takes the shape of the molecules of interest into account. The equation is especially useful in chemical engineering where phase transitions are of great importance. Since nitrogen is efficiently described by a cubic equation of state, it is expected to model accumulator gas dynamics accurately. It can be written:

$$
p=\frac{R T}{V-b}-\frac{a(T)}{V(V+b)}
$$

Where:

$$
a(T)=0.4274 \frac{R^{2} T_{c}^{2}}{p_{c}}\left[1+\kappa\left(1-\left(\frac{T}{T_{c}}\right)^{\frac{1}{2}}\right)\right]^{2}
$$

With:

$$
\kappa=0.480+1.57 \omega-0.176 \omega^{2}
$$

and:

$$
b=0.08664 \frac{R T_{c}}{p_{c}}
$$

Because the Soave-Redlich-Kwong equation is much simpler than the Benedict-Webb-Rubin equation, it is as much as six times faster to evaluate numerically.

\section{Experimental Approach}

The hydraulic set-up for the experiment is simple. A six liter piston type accumulator is connected to a proportional valve which in turn is connected to a pressure source. Pressure and temperature is measured on both sides of the accumulator. A sketch of the set-up is shown in figure 2 .

As the purpose of the research is to compare simulated data, using the thermodynamical model from section 2 to experimental data. The data has to span different temperatures and pressures. Furthermore, as the model must predict the dynamics of accumulators fitted in wind turbines, conclusions regarding validity of the model must also hold for a wide span

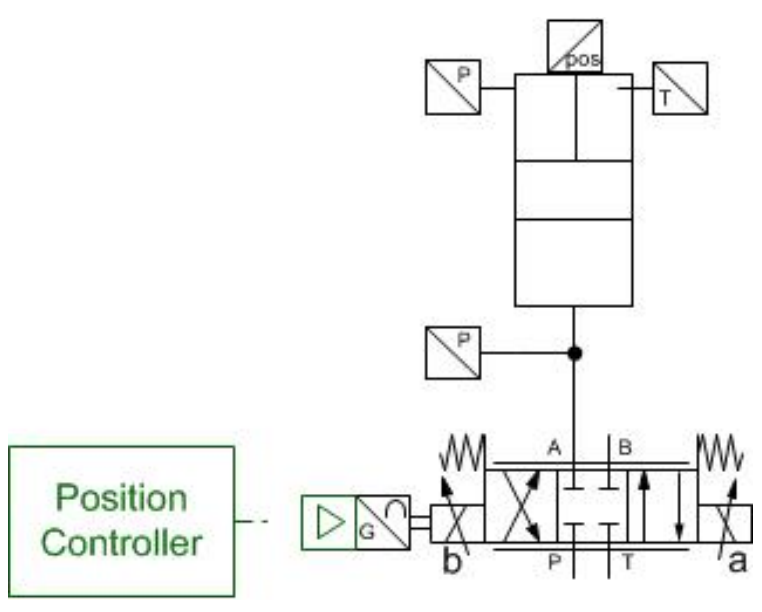

Figure 2: Overview of the experimental set-up

of ambient temperatures. The experiments conducted fall in the parameter-window illustrated in table 2 .

\begin{tabular}{|l|clc|}
\hline \multicolumn{4}{|c|}{ Maximum pressure } \\
\hline \multirow{3}{*}{ Ambient Temperature } & Low & Medium & High \\
\hline Low & H, M, L & H, M, L & H, M, L \\
Medium & H, M, L & H, M, L & H, M, L \\
High & H, M, L & H, M, L & H, M, L \\
\hline
\end{tabular}

Table 2: Parameter window. H=High, M=Medium, L=Low.

The table illustrates that all combinations of low, medium and high ambient temperatures, preload pressures and maximum pressures. This is a total of $3 \cdot 3 \cdot 3=27$ combinations. The limits of the parameters tested are listed in table 3.

\begin{tabular}{|lr|}
\hline Ambient Temperature & \\
\hline Low & $-20 \mathrm{C}^{\circ}$ \\
Medium & $20 \mathrm{C}^{\circ}$ \\
High & $60 \mathrm{C}^{\circ}$ \\
\hline Preload Pressure & \\
\hline Low & $50 \mathrm{bar}$ \\
Medium & $100 \mathrm{bar}$ \\
High & $150 \mathrm{bar}$ \\
\hline Maximum Pressure & \\
\hline Low & $180 \mathrm{bar}$ \\
Medium & $220 \mathrm{bar}$ \\
High & $250 \mathrm{bar}$ \\
\hline
\end{tabular}

Table 3: Limits of parameters tested

To obtain this parameter window, the piston was, for each of the preload pressures and ambient temperatures, moved to different minimum gas volumes. The minimum volumes were estimated using a steady state model and the ideal gas equation. Relatively small deviation on the maximum pressure from the estimated is expected. It is assumed that these small deviations does not influence the validity of the overall conclusions.

The desired ambient temperatures were reached by construct- 
ing an insulated chamber. Using solid carbon dioxide, cold ambient temperatures was obtained (table 3 gives an estimate of about $-20 \mathrm{C}^{\circ}$ ). A heat source was used for creating the high ambient temperature environment. Figure 3 shows how a well insulated heat chamber can be constructed. Pictures of the set-up are shown in figure 4.

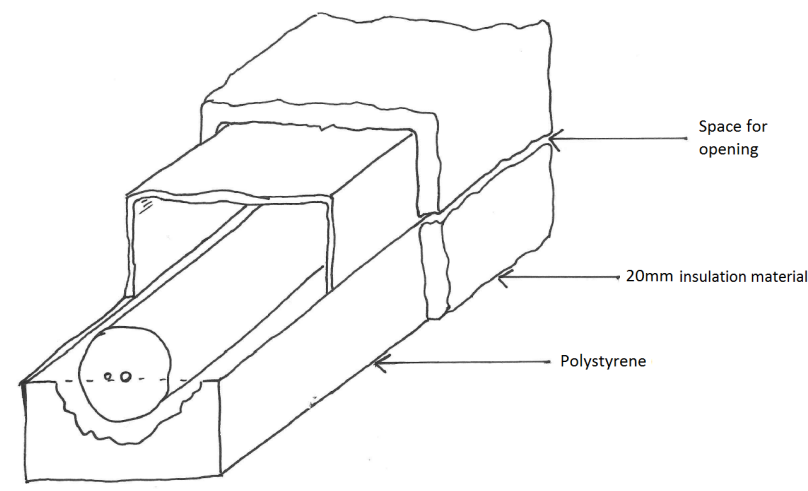

Figure 3: Sketch of how to insulate an accumulator from the environment

In order to stress the numerical models, steep pressure gradients are experimentally obtained. Practical experience has shown that it is easier to predict the behaviour at small pressure and temperature gradients than the opposite. To obtain high gradients, the gas was compressed and expanded by a realistic in application rate. An example of the expansion and compression time series is given by figure 5

In the following, the measured temperatures and pressures are plotted versus simulation results with the experimentally obtained volumes as input. In this way, it is easy to see which real gas model best fits experimental results.

\section{Results}

A limited portion of the generated results are presented. However, results from both low, medium and high ambient temperatures are shown. Also, both temperature and pressure measurements are compared to calculated results. Please note that a first order filter was used on the temperature data to limit noise. Of course this results in phase transition which should be ignored.

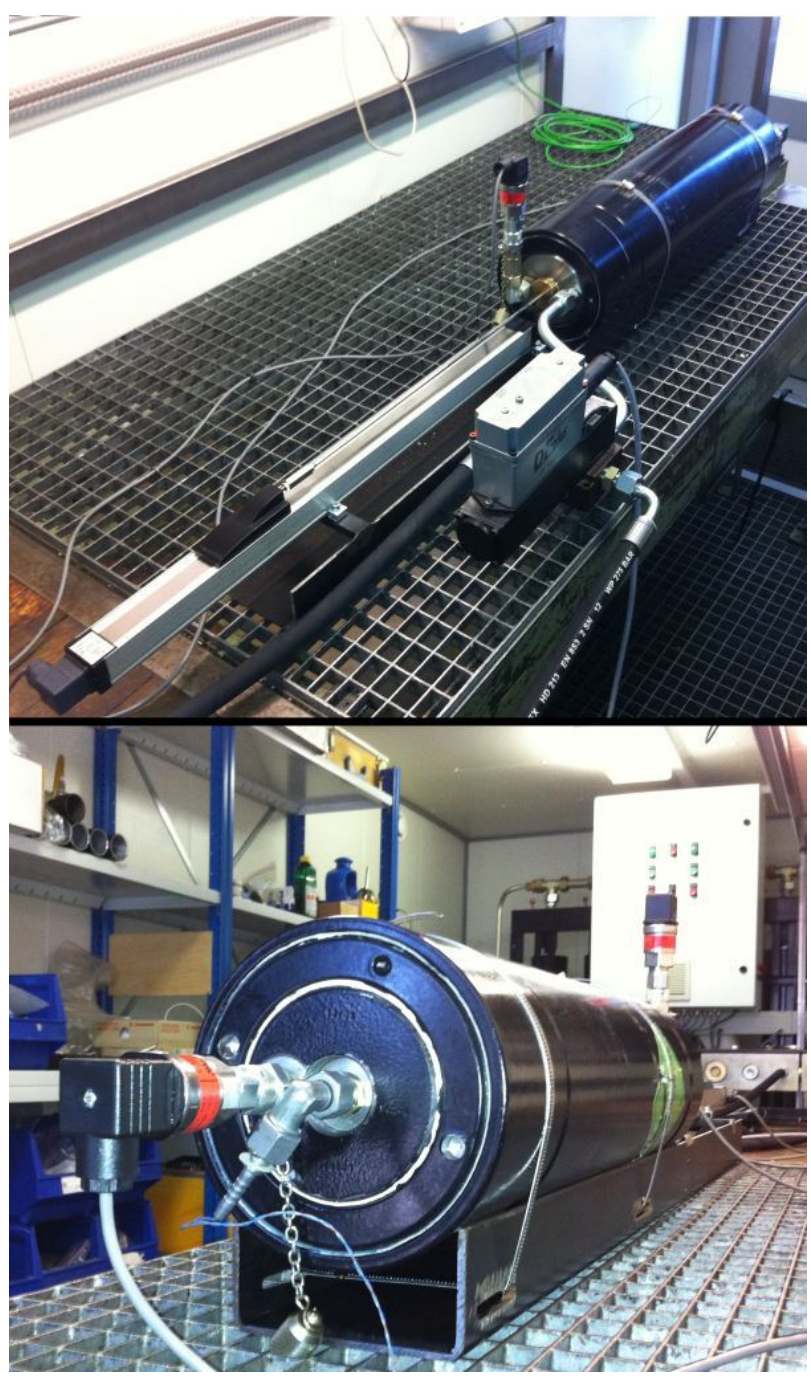

Figure 4: The setup from two angles.

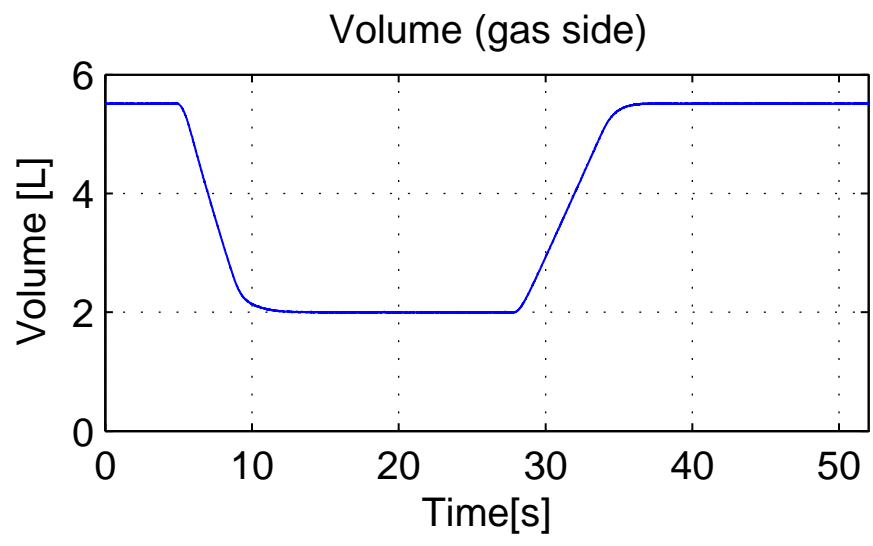

Figure 5: An overview of the experimental compression and expansion of the accumulator gas.

By driving the real gas model with the measured temperature, that is, using the data from the right hand side plots to drive the calculations in the left hand side plots, plots like figure 12 can be created. 


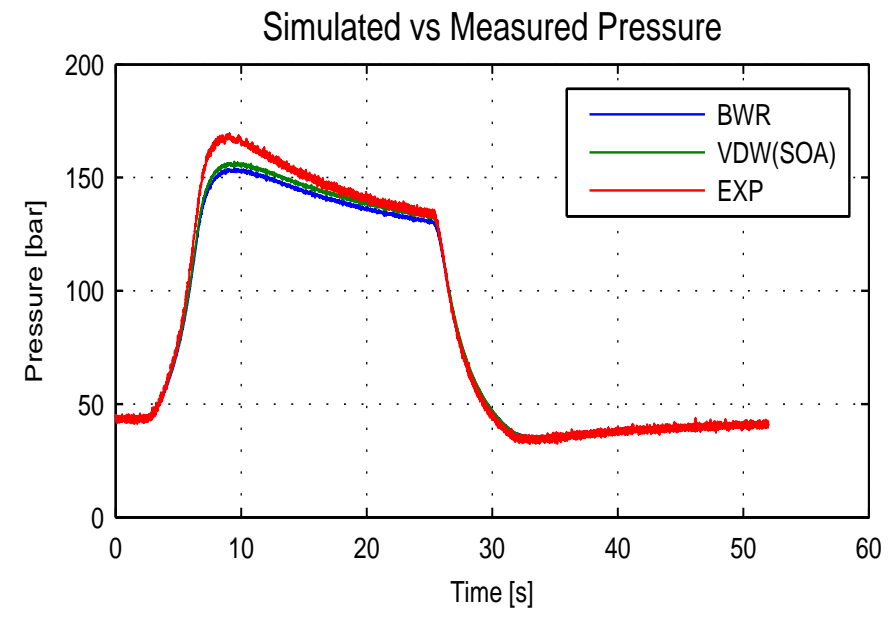

Figure 6: $p_{0}=48$ bar, $T_{0}=30^{\circ} \mathrm{C}$

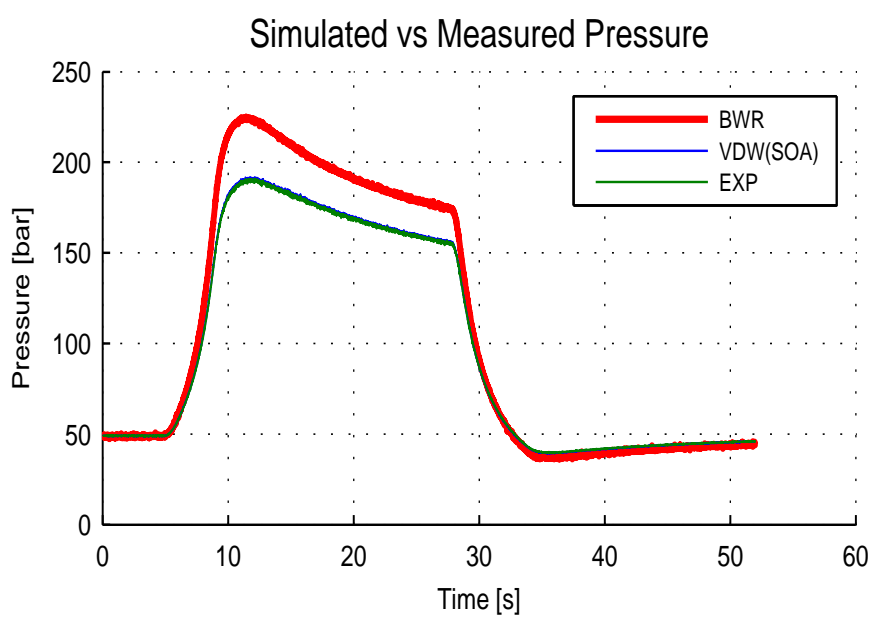

Figure 7: $p_{0}=48$ bar, $T_{0}=-5^{\circ} \mathrm{C}$

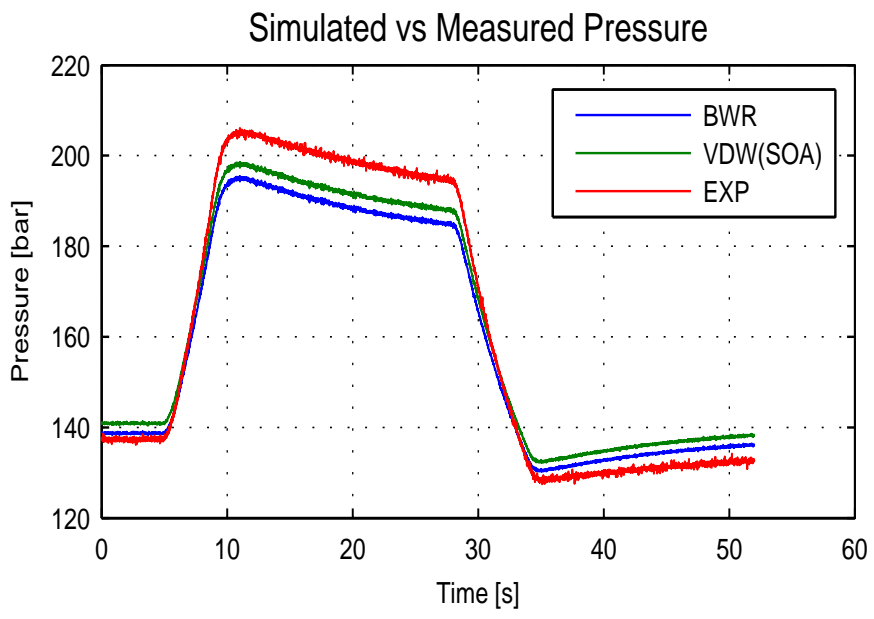

Figure 8: $p_{0}=140$ bar, $T_{0}=80^{\circ} \mathrm{C}$

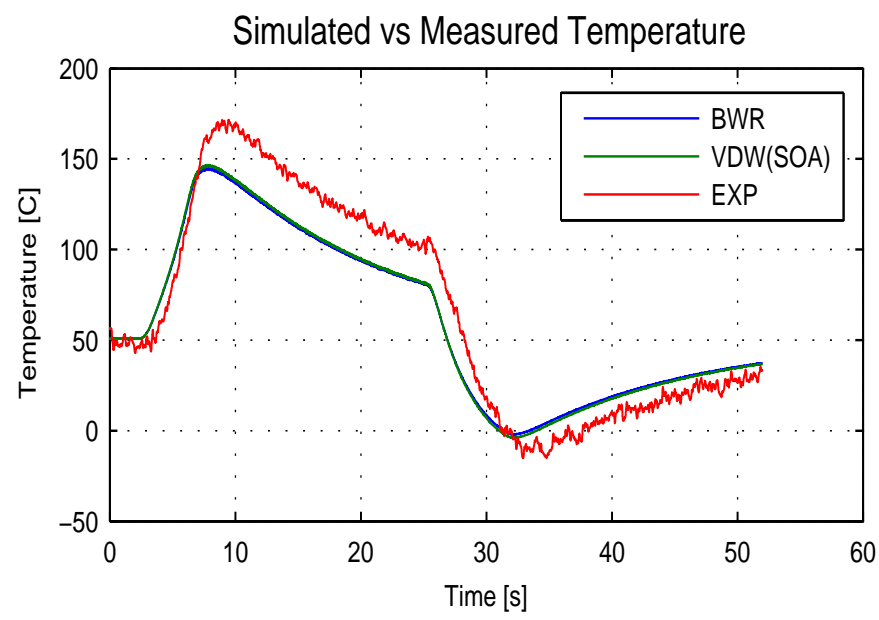

Figure 9: $p_{0}=48$ bar, $T_{0}=30^{\circ} \mathrm{C}$

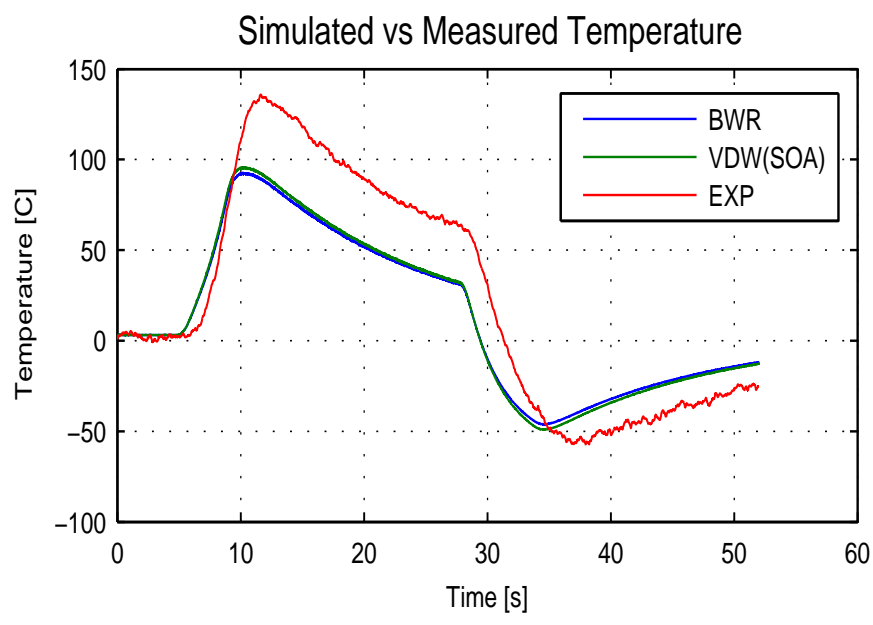

Figure 10: $p_{0}=48$ bar, $T_{0}=-5^{\circ} \mathrm{C}$

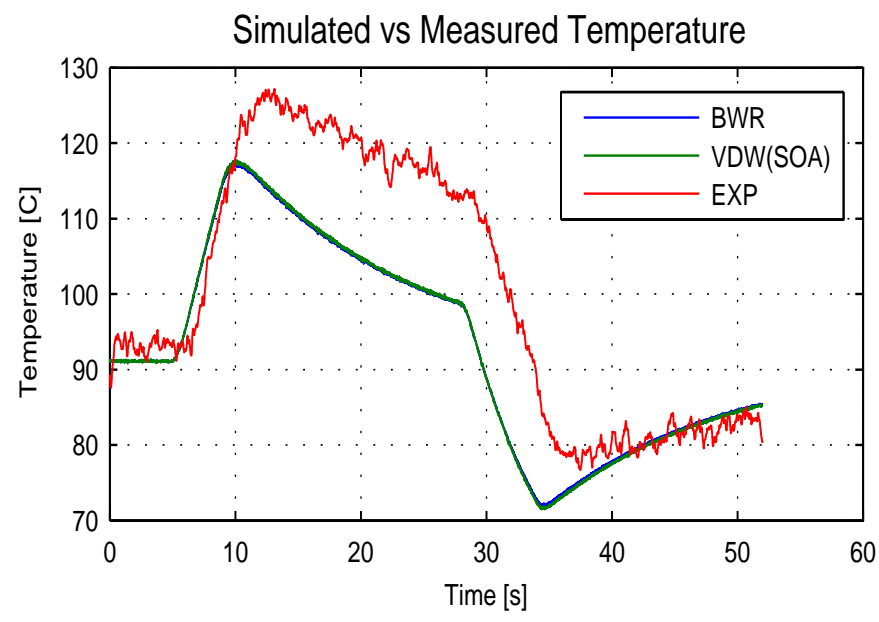

Figure 11: $p_{0}=140$ bar, $T_{0}=80^{\circ} \mathrm{C}$

\section{Discussion}

There are two clear observations from the presented results: Firstly, the two real gas models resemble in comparison to 


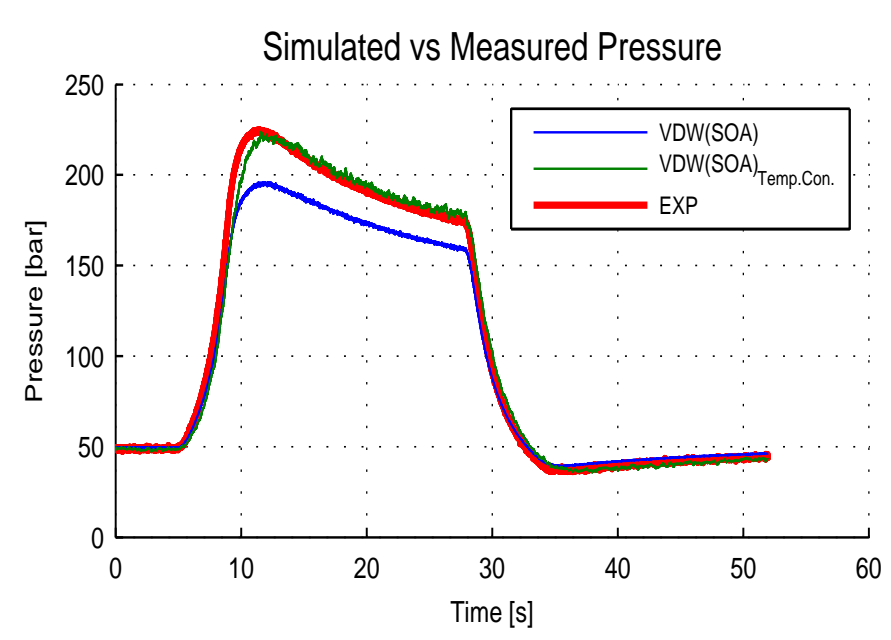

Figure 12: $p_{0}=48$ bar, $T_{0}=-5^{\circ} \mathrm{C}$, pressure calculations with calculated versus measured temperature input.

the experimental results. They both predict pressures that are some 5-10 bar lower than measured. At higher compression and expansion ratios, the calculation error is higher. Secondly, there are some clear deviations between the simulated data and the measured data. The first observation enlightens the benefits of the Soave-Redlich-Kwong equation. While maintaining the overall accuracy, it greatly reduces computation times.

The second observation requires some examination. Clearly, some error sources have to identified. Several such error sources could be suggested:

- Bad measurements

- Inaccurate real gas model

- Incorrect or inaccurate unsteady model

In the following, each of these suggestions are examined. Great effort has been put into validating and calibrating the temperature measurement devices in controlled environments prior to the research investigations. If the temperature measurements were bad despite the validation attempts, bad pressure calculations would be a result. However, as seen in figure 12 , the pressure calculations are much more accurate if the real gas model is evaluated using the measured temperature.

Figure 12 is data from the same experimental run as figure 7 It can be seen that much more accurate pressure calculations can be performed if the measured temperature is used as input to the real gas model. Similar results are obtained for all other parameters, though not presented here. This serves to validate:

- The real gas model

- The pressure measurements

- The temperature measurements
It can therefore be concluded that the primary source of error is the limited accuracy in the thermodynamical model, equation 8 . By analysing the presented data, this work serves to suggest the need for a better thermodynamical model, in order to precisely determine the dynamical behaviour of accumulators. The presented thermodynamical model has limited accuracy, because it does not model the accumulator wall. It merely suggests that the heat is transferred from the accumulator gas to the surroundings. Instead, a more precise model would employ a thick wall solution for the accumulator wall. Also, the heat conduction from the hydraulic oil to the accumulator gas (and vice versa) is not modelled. Especially when the ambient temperature are very different from the oil temperature, this will have a significant contribution.

Aside from these major issues, minor error sources in the thermodynamical model can be identified. One minor error source is the absence of the heat generated by the viscous oil in the model and also of gas flow at the accumulator wall. Especially the viscous gas flow heat contribution is a challenge to model as a function of volume change. It is likely to have negligible effect at low rate of volume change, since flow phenomena are unimportant to the problem compared to compressibility effects.

The model may be constructed by employing a heat transfer model for the accumulator wall. The model should include heat convection from the gas to the wall, heat conduction through the wall (such that temperature in the wall changes with the wall depth), heat conduction from the wall to the surroundings and also radiation from the accumulator to adjacent surfaces. Also, the model should include heat contribution from the accumulator oil by conduction though the piston to the gas. This should all serve to enlarge the insulation properties of the accumulator with respect to the gas, thus increase the peak temperature after compression. As can be seen in the results, the presented model predicts temperature- and pressure changes better after the expansion than after the compression. As accumulators are loaded during wind turbine operation at much slower rates than conducted in this experiment, the need for performance predictions here vanishes. As the accumulators empty very rapidly during emergency stops of the turbines, the crucial performance phase of the accumulator is during gas expansion. The need for a better thermodynamical model, as discussed above, may therefore have greater importance for areas in hydraulics where accumulators are loaded at high rates of volume change.

\section{Conclusion}

The presented thermodynamical model has limitations at high rates of volume changes. This is most likely due to the inaccurate modelling of the insulation properties of the accumulator. The inaccuracies are some 5-10 bar difference between measured and calculated data just after compression and expansion. The difference is higher for higher compression/expansion ratios and the difference approaches zero for infinitely slow rates of volume changes. 
A better thermodynamical model can maybe be established by implementing a heat balance on the accumulator wall in the model.

The two presented real gas models; The Benedict-WebbRubin equation and the Soave-Redlich-Kwong equation are almost similarly accurate. The Soave-Redlich-Kwong model suggests a slightly higher pressure after compression compared to the Benedict-Webb-Rubin equation. Since the Soave-Redlich-Kwong equation is much more efficient than the Benedict -Webb-Rubin equation, this work concludes that the Soave-Redlich-Kwong equation should replace the Benedict-Webb-Rubin equation in future simulations.

The quality of the measurements were confirmed by calculating the pressure using the Soave-Redlich-Kwong equation with the measured temperature, and then comparing the calculations to measured pressures. Since the calculated data matched the measured data extremely well, it is concluded that the measurements are accurate, dynamic and reliable. Also, it is concluded on this basis that the Soave-RedlichKwong real gas model is accurate enough for any application within the wind turbine technology.

\section{References}

[1] Pourmovahed A. Otis, D. R. An Algorithm for Computing Nonflow Gas Processes in Gas Springs and Hydropneumatic Accumulators. Journal of Dynamic Systems, Measurement, and Control, 107:93-96, 1985.

[2] A Pourmovahed and D. R. Otis. An Experimental Thermal Time-Constant Correlation for Hydraulic Accumulators. Journal of Dynamic Systems, Measurement and Control, 112:116-121, 1990.

[3] S. Rotthäuser. Verfahren zur Berechnung und Untersuchung hydropneumatischer Speicher. Fakultät für Maschinenwesen der Rheinisch-Westfälischen Technischen Hochschule Aachen, Essen, Germany, 1993.

[4] D. R. Otis. New Delvopment in Predicting and Modifying Performance of Hydraulic Accumulators. National Conference on Fluid Power, 1974.

[5] G. Soave. Equilibrium Constants from a Modified RedlichKwong Equation of State. Chem. Eng. Sci., 27:1197-1203, 1972. 